\title{
Surgical Treatment of Sacrococcygeal Pilonidal Sinus with a Leaf Flap
}

\author{
Jun Hyun Kim (D), Seung Min Nam (D), Eun Soo Park (D), Chang Yong Choi (D), Han Gyu Cha (D) \\ Department of Plastic and Reconstructive Surgery, Soonchunhyang University College of Medicine, Bucheon, Korea
}

\begin{abstract}
Background: Pilonidal sinus, occurring in $7 \%$ of the population, is a disease found in the cleavage of the buttocks, typically in young males after puberty. This study explored the utility of the leaf flap, a unilobed fasciocutaneous transposition flap, as a reconstructive method for treating pilonidal sinus on the sacrococcygeal area.

Methods: We present cases of eight patients with pilonidal sinus who underwent unilobed fasciocutaneous transposition flap surgery. Patients complaining of an abscess in the sacrococcygeal region were initially treated with a minimal incision to drain the abscess, and 14 days of antibiotics administration. Afterwards, the patients were re-evaluated at the outpatient clinic and scheduled for a leaf flap procedure 4 weeks after drainage. Hospitalization, the time required for full activity, and complication rates were recorded.

Results: Minor postoperative complications were observed in one patient (12.5\%). The patient required re-suturing of his wound due to an infection and wound dehiscence. The mean hospitalization was 10 days (range, 5-23 days), and the period required to return to daily activities was 4 weeks.

Conclusion: The leaf flap procedure is recommended because it is a relatively easy method for surgeons, and also because the flap has abundant perfusion. This surgical method was used as the first treatment of choice for sacrococcygeal pilonidal sinus, with the advantages of less patient discomfort, fast healing of the site and short hospitalization. Our results support the leaf flap surgery as a favored treatment for pilonidal sinus.
\end{abstract}

Keywords: Pilonidal sinus; Flap, surgical; Sacrococcygeal region

\section{Introduction}

Pilonidal sinus, typically affecting young men after puberty, is a disease occurring in approximately $7 \%$ of the entire population [1-3]. It is generally known to be an acquired affliction, while some reports describe it as congenital. Known risk factors include hair of the sacral area, poor hygiene, regional trauma, repetitive irritation and obesity [3].

As sacrococcygeal pilonidal sinus is one of the diseases that most often recurs after surgical treatment, many surgical options have been reported. According to recent literature, wide excision of the entire sinus and reconstruction with flap coverage is the preferred surgical technique, which is particularly required for large defects [4-7]. However, partial flap necrosis or loss of flap after surgery may obstruct wound healing and have a detrimental effect on both function and appearance [8-10].

The leaf flap, a unilobed fasciocutaneous transposition flap, demonstrates considerable capacity to reconstruct challenging surgical wounds, with favorable aesthetic outcomes. The abundant adjacent tissue transferred with the flap to the area of defect proves to be very effective for healing the sacrococcygeal area [11-14]. Regardless of the location, successful application of the leaf flap generally depends on correct de-

Original Article

Received: February 22, 2021

Revised: April 18, 2021

Accepted: April 20, 2021

Corresponding author: Seung Min Nam, M.D., Ph.D.

Department of Plastic and Reconstructive, Soonchunhyang University Bucheon Hospital, Soonchunhyang University College of Medicine, 170 Jomaru-ro, Wonmi-gu, Bucheon 14584, Korea

Tel: +82-32-621-5311

Fax: +82-32-621-5316

E-mail: zodiac1003@schmc.ac.kr

This is an Open Access article distributed under the terms of the Creative Commons Attribution Non-Commercial License (https://creativecommons.org/licenses/by-nc/4.0/) which permits unrestricted non-commercial use, distribution, and reproduction in any medium, provided the original work is properly cited.

(C) 2021 Korean Wound Management Society 
sign of the flap, meticulous skill of the surgeon, and of course patient consent or choice of the flap procedure itself.

The present study investigates the value of the leaf flap, a unilobed fasciocutaneous transposition flap, in the reconstruction of defects formed after treatment of sacrococcygeal pilonidal sinus.

\section{METHOD}

\section{Patients}

From July 2011 to July 2015, eight patients suffering from pilonidal sinus of the sacrococcygeal area were treated using leaf flaps at the Department of Plastic and Reconstructive Surgery of Soonchunhyang University Bucheon (Table 1). All eight patients provided informed consent. This research was approved by the Institutional Review Board of Soonchunhyang University Bucheon Hospital (IRB No. 2020-12-004) and conducted in compliance with ethical guidelines of the Declaration of Helsinki.

The subjects were treated according to a standardized protocol. Subjects with an abscess on the sacrococcygeal area initially received a minimal incision for drainage of the abscess. After the procedure, $1 \mathrm{~g}$ of amoxicillin was administered orally twice a day for 2 weeks. Additional assessment of the lesion at the outpatient clinic was performed subsequently, and the patients were scheduled for leaf flap surgery 1 month after the drainage procedure. Subjects presenting with acute infection accompanied by purulent discharge along the sinus tracts of sacrococcygeal area, without clinical abscess, were treated with a brief antibiotic therapy of $1 \mathrm{~g}$ of amoxicillin twice a day before their leaf flap surgery. Subjects presenting chronicity without acute signs of the infection underwent the surgery immediately.

The subjects were recommended to return to their normal routines after removal of the stitches, about 14 days postoperatively, and to avoid excessive physical exertion and intense sports activities for 3 to 4 weeks. The patients visited the outpatient clinic once a month for follow-up for the first 6 months, after which they visited once every 6 months for the following year.

\section{Surgical technique}

As the surgery was to reconstruct a perianal defect, the operation began with general or regional (spinal) anesthesia. The patient was placed in a prone position and both buttocks were prepared with sufficient exposure of the perianal defect. After sterilizing the skin with $10 \%$ povidone-iodine, and antibiotic prophylaxis for wound infection, the tissue to be excised was marked, and using gentian-violet soaked wooden chalk, the design of the flap was drawn on the skin, with the portion that was to be inserted into the defect after de-epithelization marked with slashes, resembling the veins of a leaf (Fig. 1). Between 0.5 and $1 \mathrm{~mL}$ of methylene blue dye was injected into the most conspicuous opening of the sinus, taking care not to apply excessive pressure, to better define the afflicted tissue. After a 1:100,000 epinephrine and 2\% lidocaine solution was injected into the designed incision line, a wide vertical excision was made including the post-sacral fascia, using a No. 15 surgical blade, Metzenbaum scissors and electrocautery, thereby removing entire tracts en bloc. Hemostasis was then induced with an electrical coagulator. Either a left or right-sided leaf flap, containing the gluteal fascia, was designed and tailored to

Table 1. Characteristics of patients

\begin{tabular}{|c|c|c|c|c|c|}
\hline Patient & $\begin{array}{l}\text { Sex/age } \\
\text { (yr) }\end{array}$ & Diagnosis & Treatment & $\begin{array}{l}\text { Defect size after excision } \\
(\mathrm{cm})\end{array}$ & Complication \\
\hline 1 & $\mathrm{~F} / 76$ & Pilonidal sinus & Leaf flap & $9.2 \times 5.1$ & № \\
\hline 2 & $\mathrm{~F} / 78$ & Pilonidal sinus & Leaf flap & $9.0 \times 5.3$ & № \\
\hline 3 & M/35 & Pilonidal sinus with acute abscess & Leaf flap and drainage procedure & $10.0 \times 5.9$ & Wound dehiscence \\
\hline 4 & $\mathrm{~F} / 18$ & Pilonidal sinus & Leaf flap & $8.4 \times 4.8$ & No \\
\hline 5 & $\mathrm{M} / 16$ & Pilonidal sinus & Leaf flap & $8.8 \times 4.7$ & No \\
\hline 6 & $M / 28$ & Pilonidal sinus with acute infection & Leaf flap & $9.7 \times 5.6$ & No \\
\hline 7 & $M / 36$ & Pilonidal sinus & Leaf flap & $9.5 \times 5.1$ & No \\
\hline 8 & $\mathrm{M} / 29$ & Pilonidal sinus with acute infection & Leaf flap & $9.8 \times 5.7$ & No \\
\hline Mean & 52.5 & & & $9.3 \times 5.3$ & \\
\hline
\end{tabular}

F, female; M, male. 


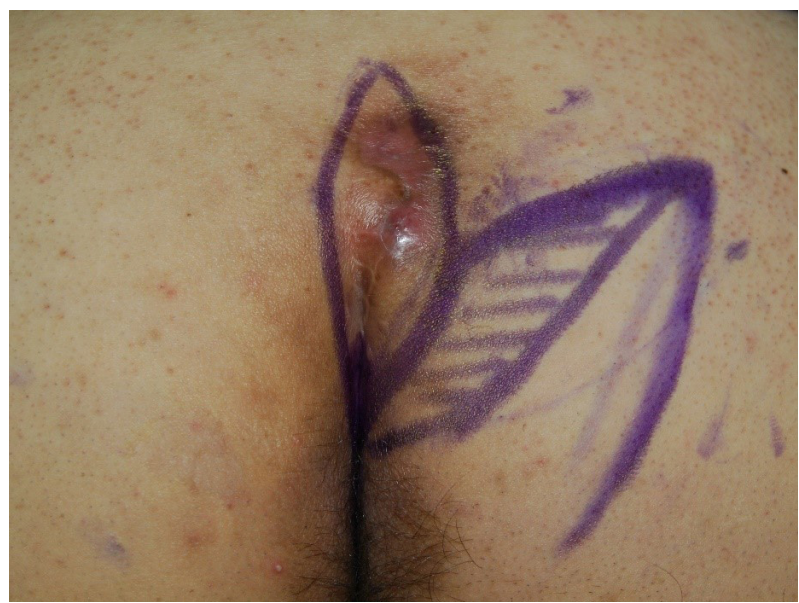

Fig. 1. The design of the leaf flap. The shaded area of the flap design is de-epithelized.

be $20 \%-25 \%$ larger than the defect size after excision, and to be rotated approximately $60^{\circ}$ to cover the defect area. Afterwards, the flap was elevated on its inferior edge for sufficient mobilization and was transposed medially to fill the defect made after the excision, without tension or any dead space. A Jackson-Pratt drain was inserted in the wound cavity and the subcutaneous layer was repaired with 4-0 Maxon interrupted sutures and the skin was closed with 4-0 nylon vertical mattress sutures (Fig. 2). Antibiotics were administered for 5 days postoperatively, initially intravenously and later orally to prevent perianal wound infection. The Jackson-Pratt drain was removed when the drain fluid fell below $10 \mathrm{~mL}$. Total stitch out was performed on the 14th postoperative day (Fig. 3).

\section{RESULTS}

The mean age of the eight subjects was 52.5 years (range, 1678 years), and five of the eight patients were male. One patient reported with an abscess initially and was treated first with an open procedure. Though two patients in this study had infections, they were deemed ready for the surgical procedure after a short period of antibiotic therapy.

Minor postoperative complications occurred in one patient. The patient required wound re-suture because of an infection and wound dehiscence.

The mean hospitalization for the patients was 10 days (range, 5-23 days), and after an average of 4 weeks, the subjects could return to their daily lives.
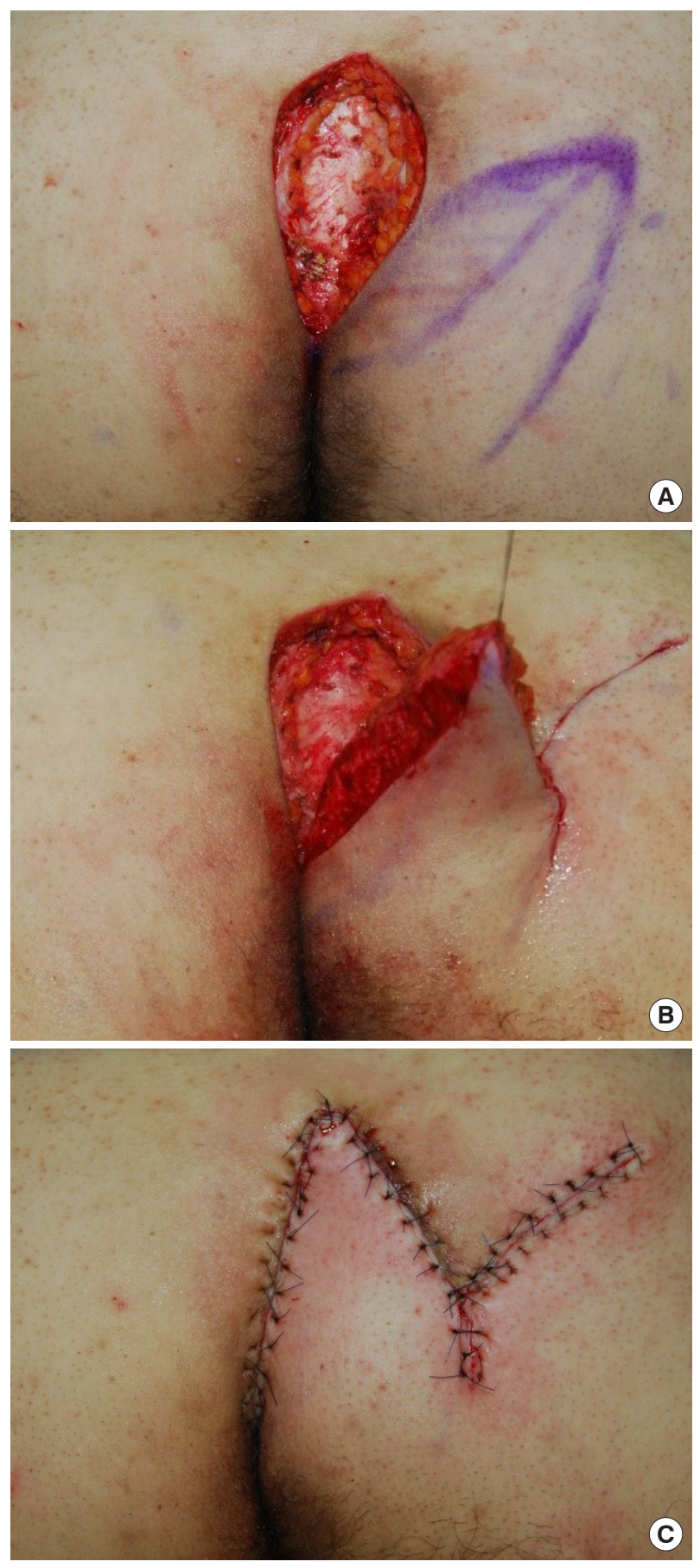

Fig. 2. Intraoperative photographs. (A) Defect formed after complete excision of the pilonidal sinus. (B) Elevated leaf flap. The previously shaded area was de-epithelized to minimize contour deformity after flap inset. (C) Immediate postoperative photographic finding.

\section{DISCUSSION}

The direct cause of pilonidal sinus on the sacrococcygeal area 

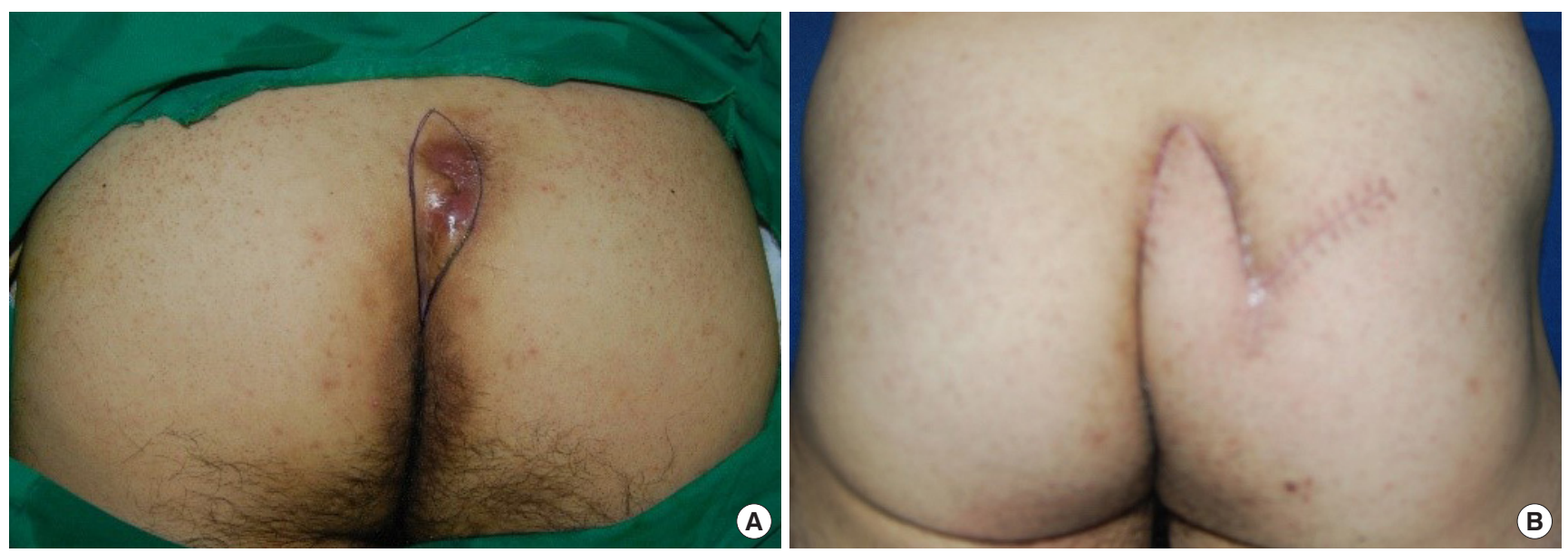

Fig. 3. Preoperative and long-term follow-up photographic findings. (A) Preoperative photographic finding of pilonidal sinus. Abscess formation was accompanied in this 35-year-old male patient. (B) Four weeks postoperative photographic finding.

has not been identified. However, hair insertion into the skin, followed by formation of foreign body granuloma, is believed to be the most likely cause [15-17]. Karydakis [16] emphasized three key factors contributing to this process. The first is the invading foreign body; namely, hair. Second is the external force caused by the narrow cleft which causes the insertion of the hair, and the last is the weakness of the raphe. Cubukcu et al. [18] suggested that body mass index can be a risk factor that contributes to early onset and worsens the course of the disease.

While the general consensus for treating pilonidal sinus is that the surgeon should completely remove the sinus, a "gold standard" method for reconstruction is yet to be established [19-21]. Surgical procedures are classified into three types; the first is the open technique, the second is the midline and offmidline or closing with asymmetry and the last is flap surgery [22]. Lee et al. [19] argued for only excising in early stages of the disease and a reconstructive procedure with flap for recurrent sinus. McCallum et al. [23] recommended off-midline closure as the treatment of choice for pilonidal cyst and sinus of the sacrococcygeal area when the optimal surgical option is primary closure. Open procedures are conventional techniques inducing secondary intention healing and report a low recurrence. However, they require more time and effort for healing [20]. In a study dealing exploring the open procedure technique for 65 patients, the mean healing period was $43 \pm 10.4$ days [24]. On the other hand, while procedures using midline closure have the advantage of rapid healing, they also have the shortcomings of high rates of recurrence or wound problems. It was reported that 16 out of 218 (7.3\%) subjects who received open procedure experienced complications of morbidity versus 26 out of 215 (12.1\%) subjects who received midline closure [23]. Allen-Mersh [25] suggested that the difference among these three techniques is that a midline closure procedure does not alter the three factors of Karydakis, while open procedures change the contour of the cleft and decrease both local invasion of debris and irritation to the sacrococcygeal area. Off-midline closure is based on his suggestion. Therefore, by removing the vulnerable raphe and replacing it with normal tissue the midline scar at the depth of the natal cleft and hair insertion can be prevented [15-17,22].

Flap procedures can remove more of the raphe compared to earlier methods, flattening the contour of the natal cleft, reducing depth and repositioning hair follicles, thereby reducing the three risk factors of Karydakis [7,9]. These procedures, including a variety of flap techniques such as V-Y advancement, Z-plasty and rhomboid shape flaps, improved early-stage outcomes and lowered the recurrence rates [19]. In contrast, several authors have questioned the efficacy of these techniques, suggesting that flap techniques are more challenging than the open technique and that they have significant rates of flap loss or loss of skin sensation. These authors argue that the flap procedures should be used to reconstruct only recurrent or complex pilonidal sinus $[9,19,22]$.

Some single institution studies have demonstrated that the flap techniques provide abundant vascularity, with low flap necrosis rates and lower morbidity and relapse rates [6-9]. The leaf flap, a unilobed fasciocutaneous flap, is a modified form of the Dufourmentel flap. In the leaf flap procedure, there are three advantages. Firstly, a wider base can be observed in the 
leaf flap compared to that of the Limberg flap or the rhomboid transposition flap; therefore the leaf flap has more robust vascularity. Secondly, the tip of the leaf flap is more appropriately located than in the Dufourmentel rhomboid flap, and the angle of the triangular shaped flap is less blunt; the leaf flap thus allows easy transposition of the flap. Finally, we did not fix the flap to the sacrococcygeal fascia, which produced both better flattening of the cleft and rearrangement of the position of the hair follicles.

In several reports, wound closure of the flaps were done by suturing in three layers, repairing the base of the flap to the fascia of that area; some used a compressive wound dressing instead of a drain $[7,9,22]$. However, we did not fix the flap to the sacrococcygeal fascia as descripted above, instead maintaining a suction drain for an extended period to successfully prevent seroma formation, the most common complication of the flap procedure [17,22].

In this study, none of the patients reported dissatisfaction with the aesthetic aspect. Most patients went home within 10 postoperative days and were able to sit more quickly and return to work earlier. Postoperative complications were rare, with only one patient requiring re-suturing. This case had presented with a particularly severe infection due to preoperative abscess formation. Though all infected areas were excised, the infection recurred postoperatively, resulting in wound dehiscence. The patient's case demonstrates that if the pilonidal sinus is accompanied with infection, it is necessary to control the infection thorough antibiotics and drainage before the operation, and to perform sufficient resection during the operation. The loss of skin sensation was negligible.

Among numerous treatment methods for pilonidal sinus of the sacrococcygeal area, is prone to postoperative recurrence, the leaf flap can be an attractive option because it is a relatively easy procedure for plastic surgeons, and also because the flap provides abundant perfusion.

\section{Conflict of interest}

No potential conflict of interest relevant to this article was reported.

\section{ORCID iDs}

Jun Hyun Kim

Seung Min Nam

Eun Soo Park https://orcid.org/0000-0003-0410-2680

https://orcid.org/0000-0001-7865-5013

https://orcid.org/0000-0003-2966-9122
Chang Yong Choi

Han Gyu Cha

https://orcid.org/0000-0002-6385-0817

https://orcid.org/0000-0003-3059-1334

\section{References}

1. Armstrong JH, Barcia PJ. Pilonidal sinus disease: the conservative approach. Arch Surg 1994;129:914-7.

2. Sondenaa K, Nesvik I, Andersen E, et al. Recurrent pilonidal sinus after excision with closed or open treatment: final result of a randomised trial. Eur J Surg 1996;162:237-40.

3. Surrell JA. Pilonidal disease. Surg Clin North Am 1994;74: 1309-15.

4. Cihan A, Ucan BH, Comert M, et al. Superiority of asymmetric modified Limberg flap for surgical treatment of pilonidal disease. Dis Colon Rectum 2006;49:244-9.

5. Ertan T, Koc M, Gocmen E, et al. Does technique alter quality of life after pilonidal sinus surgery? Am J Surg 2005; 190:388-92.

6. Eryilmaz R, Sahin M, Alimoglu O, et al. Surgical treatment of sacrococcygeal pilonidal sinus with the Limberg transposition flap. Surgery 2003;134:745-9.

7. Mahdy T. Surgical treatment of the pilonidal disease: primary closure or flap reconstruction after excision. Dis Colon Rectum 2008;51:1816-22.

8. Akin M, Gokbayir H, Kilic K, et al. Rhomboid excision and Limberg flap for managing pilonidal sinus: long-term results in 411 patients. Colorectal Dis 2008;10:945-8.

9. el-Khadrawy O, Hashish M, Ismail K, et al. Outcome of the rhomboid flap for recurrent pilonidal disease. World J Surg 2009;33:1064-8.

10. Unalp HR, Derici H, Kamer E, et al. Lower recurrence rate for Limberg vs. V-Y flap for pilonidal sinus. Dis Colon Rectum 2007;50:1436-44.

11. Borges AF. The rhombic flap. Plast Reconstr Surg 1981; 67:458-66.

12. Bray DA. Clinical applications of the rhomboid flap. Arch Otolaryngol 1983;109:37-42.

13. Fee WE Jr, Gunter JP, Carder HM. Rhomboid flap principles and common variations. Laryngoscope 1976;86:170611.

14. Larrabee WF Jr, Trachy R, Sutton D, et al. Rhomboid flap dynamics. Arch Otolaryngol 1981;107:755-7.

15. Bascom J. Pilonidal disease: origin from follicles of hairs and results of follicle removal as treatment. Surgery 1980; 87:567-72.

16. Karydakis GE. Easy and successful treatment of pilonidal 
sinus after explanation of its causative process. Aust N Z J Surg 1992;62:385-9.

17. Bessa SS. Results of the lateral advancing flap operation (modified Karydakis procedure) for the management of pilonidal sinus disease. Dis Colon Rectum 2007;50:193540.

18. Cubukcu A, Gonullu NN, Paksoy M, et al. The role of obesity on the recurrence of pilonidal sinus disease in patients, who were treated by excision and Limberg flap transposition. Int J Colorectal Dis 2000;15:173-5.

19. Lee PJ, Raniga S, Biyani DK, et al. Sacrococcygeal pilonidal disease. Colorectal Dis 2008;10:639-50.

20. Soll C, Hahnloser D, Dindo D, et al. A novel approach for treatment of sacrococcygeal pilonidal sinus: less is more. Int J Colorectal Dis 2008;23:177-80.

21. Tezel E, Bostanci H, Anadol AZ, et al. Cleft lift procedure for sacrococcygeal pilonidal disease. Dis Colon Rectum 2009;52:135-9.

22. Lieto E, Castellano P, Pinto M, et al. Dufourmentel rhomboid flap in the radical treatment of primary and recurrent sacrococcygeal pilonidal disease. Dis Colon Rectum 2010; 53:1061-8.

23. McCallum IJ, King PM, Bruce J. Healing by primary closure versus open healing after surgery for pilonidal sinus: systematic review and meta-analysis. BMJ 2008;336:868-71.

24. Kement M, Oncel M, Kurt N, et al. Sinus excision for the treatment of limited chronic pilonidal disease: results after a medium-term follow-up. Dis Colon Rectum 2006;49:175862.

25. Allen-Mersh TG. Pilonidal sinus: finding the right track for treatment. Br J Surg 1990;77:123-32. 